\title{
How to utilize tacit knowledge in health organizations: An Iranian perspective
}

\author{
Ensiyeh Jamshidi ${ }^{1}$, Sima Nedjat*2 $^{2}$, Saharnaz Nedjat ${ }^{3}$, Sima Nikooee ${ }^{2}$, Narges Rostamigooran ${ }^{4}$, \\ Reza Majdzadeh ${ }^{5}$
}

\begin{abstract}
Background: Studies show that $90 \%$ of an organization's knowledge is embedded and synthesized in its employees' minds. Thus, when employees leave the organization or their positions change, their valuable knowledge, skills, and experiences are lost, however, if used properly, tacit knowledge can be a source of innovation and competitive advantage in an organization. This study aimed at exploring the methods for sharing and utilizing tacit knowledge in health organizations.

Methods: In this study, qualitative approach was adopted to explore ways of utilizing tacit knowledge in health organizations. Tacit knowledge experts, who had published at least one relevant article, conducted 17 individual and 2 group interviews. Purposeful sampling was used to select the participants. Methods for sharing and utilizing tacit knowledge were explored by holding in-depth semi-structured interviews. Data were analyzed using thematic analysis.

Results: The results were summarized into 5 categories and 18 themes. The categories included 'identification of different dimensions of organizational knowledge', 'prerequisites of tacit knowledge utilization', 'defining the process of tacit knowledge utilization', 'converting tacit to explicit knowledge', and 'converting tacit to tacit knowledge'. Participants believed that the process of converting tacit to explicit knowledge was a cyclical process that included the understanding the existing situation and detecting knowledge entry points, identifying knowledge items and harvesting them, assessment, codification, and standardization, entry into knowledge repository, and updating.

Conclusion: Our results revealed that health organizations need the prerequisites of tacit knowledge sharing to acquire the capacity to utilize this kind of knowledge. Because the themes extracted in this study are rarely used in health organizations, the results will be helpful in guiding the development of knowledge utilization strategies and planning in these organizations.
\end{abstract}

Keywords: Knowledge utilization, Tacit knowledge, Health organizations

Conflicts of Interest: None declared

Funding: Knowledge Utilization Research Center of Tehran University of Medical Sciences, Tehran, Iran

\section{*This work has been published underCC BY-NC-SA 1.0 license.}

Copyright $₫$ Iran University of Medical Sciences

Cite this article as: Jamshidi E, Nedjat S, Nedjat S, Nikooee S, Rostamigooran N, Majdzadeh R. How to utilize tacit knowledge in health organizations: An Iranian perspective. Med J Islam Repub Iran. 2018 (22 Nov);32:116. https://doi.org/10.14196/mjiri.32.116

\section{Introduction}

Organizational knowledge translation (KT) strategies are believed to lead to more effective services in improving public health. Unfortunately, KT strategies have not led to the expected achievements in evidence-based decision-

Corresponding author:DrSima Nedjat, nejat_s@farabi.tums.ac.ir; sima.nedjat2011@gmail.com

1. Community-based Participatory Research Center, Iranian Institute for Reduction of High-Risk Behaviors, Tehran University of Medical Sciences, Tehran, Iran

2. Knowledge Utilization Research Center, Tehran University of Medical Sciences, Tehran, Iran

3. Epidemiology and Biostatistics Department, School of Public Health, Knowledge Utilization Research Center, Tehran University of Medical Sciences, Tehran, Iran

4. Secretariat of Supreme Council of Health and Food Security, MOH \& ME

5. School of Public Health and Knowledge Utilization Research Center, Tehran University of Medical Sciences, Head of Iran's National Institute of Health Research, Tehran, Iran making yet (1). Based on the epistemology of logical empiricism which emphasizes causal relations, the role of tacit form of knowledge in the KT process is undermined, while social constructivism has an interaction-based view of knowledge translation and considers people's experiences,

$\uparrow$ What is "already known” in this topic:

Tacit knowledge is similar to a black box of an organization. It cannot be easily transferred, but if it were transferred, it would confirm the sustainability of knowledge in the organization.

\section{$\rightarrow$ What this article adds:}

The present study revealed that organizations should change their construction and execution to obtain the capacity to utilize tacit knowledge. Participants of this study believed that converting tacit knowledge into explicit knowledge can be done through flowcharts, instructions, organizational processes, and duties. 
facts, and understanding as socially constructed through interactions $\{$ Lincoln Y, \#2\} (2). Therefore, in this paradigm, knowledge is formed in its interactions with individuals.

Explicit knowledge is about 'what' and tacit knowledge is about 'how'. Explicit knowledge is documented in formal language, print or electronic media, while tacit knowledge is experience-based, formed on the basis of practice, and is not easily expressed (3). Tacit knowledge includes different dimensions and is the result of professional experiences and mutual understanding in interactions with others (teams of colleagues or stakeholders). Unfortunately, this dimension of knowledge has not gained equal attention as explicit knowledge with the growth of evidence-based medicine in logical empiricist epistemology $(4,5)$.

Studies show that $90 \%$ of an organization's knowledge is embedded and synthesized in its employees' minds and not documented. Thus, when employees leave the organization or their positions change, their valuable knowledge, skills, and experiences are lost (3), whereas, if used properly, tacit knowledge can be a source of innovation and competitive advantage in offering high quality services $(6,7)$. The process of tacit knowledge sharing helps an organization to move forward in ways different from its competitors and based on its own valuable, scarce, limited, and inimitable resources(8).

In Nonaka and Takeuchi's organizational knowledge creation theory, knowledge is described as a continuum. At one end of this continuum is 'absolute tacit knowledge' and at the other end is 'absolute explicit knowledge'. Most of human knowledge falls between these 2 ends and includes both tacit and explicit knowledge (8).

Tacit knowledge is important but not easy to access. This kind of knowledge is similar to a black box to top managers of an organization because it is intuitive and difficult to express and display (9). Moreover, it cannot be easily transformed into explicit knowledge, but if it were transformed, it would guarantee the sustainability of knowledge in that organization (10).

Different studies have referred to several techniques for eliciting tacit knowledge, which are as follow: using information technology, customization, consulting committees, changing working shifts, storytelling, working groups (3), holding workshops and interorganizational and intraorganizational seminars based on problem solving, using knowledge facilitators (11), on-job training, internship, increasing social interactions in the workplace, working groups, and informal networks, etc. (11). In different societies, however, given their diverse infrastructures and organizational cultures, various mechanisms are expected to apply in sharing and utilizing tacit knowledge.

In health organizations and institutions, the role of tacit knowledge in organizational knowledge management has not yet been fully understood. This deficiency is evident in the rapid changing of managers -that is more evident in developing countries-, not using qualified experts in their final working years or after retirement, and lack of required mechanisms for sharing tacit knowledge. As a result, knowledge is not accumulated in the organization and resources are wasted for production and replacement of knowledge capital.

Furthermore, practitioners in public healthcare services work in a complex environment, where formal research literature cannot be accessed. In these environments, tacit knowledge can help in the understanding of explicit knowledge (12) and can complement technical skills for health care delivery (13). Research results have also confirmed that health practitioners lay more emphasis on tacit knowledge while tackling health problems $(14,15)$. Studies have also reported the crucial role of tacit knowledge in team-based practice $(12,16,17)$ and collective decisionmaking (16). The importance of strengthening teamwork interactions and discussions for collective decision- making is inevitable for organizations in developing countries, where organizational members may be reluctant to collaborate or share information with others to maintain their dominant position or to be acknowledged by their superiors. This spirit is damaging to group cohesiveness (18). Thus, providing a framework for sharing tacit knowledge and improving the knowledge creation cycle is highly important for tacit and explicit knowledge conversion processes and for promoting collective decision- making. To our knowledge, few studies have been conducted on the methods of utilizing tacit knowledge in health organizations in these countries. Therefore, this study explored the solutions to share and utilize tacit knowledge in health organizations.

\section{Methods}

Qualitative approach was used to explore different methods of utilizing tacit knowledge in health organizations by using a semi-structured interview guide that was handed to the participants. Tacit knowledge experts, who were Iranian academics publishing peer reviewed articles on the subject of tacit knowledge in the last 5 years were included in the study through purposeful sampling. Their viewpoints on methods of sharing and utilizing tacit knowledge were explored through semi-structured interviews. Interviews revolved around methods of utilizing knowledge in health organizations.

On the whole, 17 individual and 2 group interviews (8 individuals in each group) were conducted. After obtaining an oral informed consent from the participants, we collected data and continued until data saturation. Interviews were conducted at the participants' workplaces or at the research center's conference hall, based on their preferences. In every interview, one person who took notes accompanied the facilitator. Every interview lasted between 45 to 60 minutes. The facilitator introduced herself according to the initial protocol and briefly discussed the research objectives. Based on the interviewees' responses during the interviews and analyses conducted during these interviews, other questions were added to the initial interview guide. All interviews were recorded and all audio files were saved, transcribed, and codified. Data included audio files, recorded interviews, facilitator's and note-taker's notes during interviews, and transcriptions. Analysis was done using thematic analysis. The stages of data analysis were as follow: documenting the interviews; studying typed files care- 
fully and repeatedly; and if required, listening to the interviews; open coding; axial coding; and detecting the relations between these axes, and selective coding.

To increase the trustworthiness of the study, in addition to the coding done by the facilitator, another researcher codified $10 \%$ of the interviews independently and the results were compared with each other. To reach a consensus about the disagreements over codified issues, a meeting was arranged and a final agreement was reached. Moreover, the researcher analyzed and explored the initial categories following initial data gathering. Then, based on the results of analysis, data were gathered again in the following interviews. The interviews' data were managed via MaxQDA10 software.

\section{Results}

The participating specialties by number were as follow:7 knowledge management experts, 2 epidemiologists, 1 Health education and promotion expert, 2 preventive medicine experts, 3 health management and information experts, 2 medical science and informatics experts.

Participants laid emphasis on the necessity of sharing knowledge and its dynamic nature in the organization. One of the participants said,

"Knowledge management aims to enable us to enter knowledge into a cycle to avoid its stagnancy." (p.3, Knowledge management expert).

Regarding methods for utilizing tacit knowledge, based on the discussions, 5 categories and 18 themes were elicited (Table 1).

Each of the categories and relevant themes is explained below, and the exact phrases stated by participants have been quoted in italic form.

\section{Identifying different dimensions of organizational knowledge}

This category included individual and collective dimensions of organizational knowledge, as explained below:

Tacit knowledge is utilizable in individual and collective dimensions. One of the participants noted,

"The individual dimension of tacit knowledge is defined as the flowing, applied, practical, voluntary, and specific knowledge of the individual; its translation is through individual learning by role playing or learning-by-doing .... The structure of tacit knowledge includes organizational routines, working experiences, common norms, communications, coordination, etc. We have learning organizations. This knowledge can be translated by networking on which organizations should be emphasized." (p.16, Medical science and informatics expert).

\section{Prerequisites of tacit knowledge utilization}

Emerging codes of this category mentioned in Table 1 and explained below:

Developing a knowledge utilization program: The status quo, vision, needs, limitations, and resources must be laid down in this program. Tacit knowledge management requires a specific goal. The reasons for not utilizing knowledge will be examined and solutions with maximum profit and minimum loss will be predicted.

Foreseeing a flexible structure: A prerequisite of knowledge utilization is the suitability of knowledge to the structure of the organization, which in turn determines the nature of knowledge dissemination. From the perspective of the participants, knowledge sharing is facilitated when the infrastructures of knowledge management are included in both the organizational structures and organizational performance and incorporated into the assessment of individual and organizational performance. In this regard, one of the participants declared,

"When the subject of knowledge management is discussed in the programs of bureaucratic transformation such as the 'five-year development program' etc., healthcare organizations tend to move toward knowledge sharing. When it is specified as an organization's duty and we train people and give them the needed tools, we can then evaluate them and consider these in their promotion and pay rise. However, tacit knowledge management is not included as an obligation in organizational processes. Therefore, it is a trend that will easily move into oblivion...." (p.14, Health management and information expert)

Table 1. Categories and themes extracted from the interviews

\begin{tabular}{l} 
Category \\
\hline Identifying different dimensions of organizational knowledge \\
Prerequisites of tacit knowledge utilization
\end{tabular}

Prerequisites of tacit knowledge utilization

Defining the process of tacit knowledge utilization
Themes

Individual and collective dimensions of organizational knowledge

Developing a knowledge utilization program

Foreseeing a flexible structure

Establishing an organizational culture for knowledge sharing

Considering an organizational unit or knowledge workers for utilizing tacit knowledge Developing a performance incentives system

Managerial support

Understanding the existing situation and detecting knowledge entry points

Identifying knowledge items

Harvesting knowledge items

Assessment, codification, and standardization of harvested knowledge items

Entering standardized knowledge items into the organizational process and knowledge repository

Updating

Converting tacit to explicit knowledge

Documenting and recording the experiences of experts and pioneers and lessons learned

Utilizing digital media and virtual electronic space for sharing ideas

Storytelling

Apprenticeship, mentoring, Learning by doing 
From the perspective of the participants, increased freedom and control at the individual level call for an autonomous organization that allows the internalized knowledge to become operational and is reflected in one's behavior. The participants believed that the internalization of knowledge required proper structures as 'learner organizations'.

\section{Establishing an organizational culture for knowledge sharing}

Establishing an organizational culture of knowledge sharing entails not just an effort on creating tools, but also a huge social effort and activity on cultural institutions. From the participants' perspectives, an increase in the level of self-management in a decentralized organization leads to the internalization of knowledge in such a way that it becomes a part and parcel of an organization's culture, which in turn causes its stability. Using training and not teaching methods, which is a reciprocal approach, also contributes to the internalization of knowledge. According to one of the participants,

"Hierarchical organizational structures are not effective when we are dealing with tacit knowledge. The structure of the organization must resemble a matrix; it must be agile, decentralized, and be able to change constantly. They must also be able to deploy the individuals in different posts and positions. This organization is dynamic and flexible and the learner transfers the tacit knowledge, which includes organizational routines, working experiences, common norms, communication, coordination etc., through networking." (p.16, Medical science and informatics expert)

Considering an organizational unit or knowledge workers for utilizing tacit knowledge

Some of the participants proposed appointing knowledge brokers to facilitate knowledge exchange in the organization. Setting a common language or an interpreter's mediation in extracting knowledge, especially technical and specialized knowledge, was among the participants' proposals.

\section{Developing a performance incentives system}

When the benefits of knowledge sharing become clear, it will encourage individuals to take part in knowledge sharing processes. The most important consequences of knowledge sharing for individuals are the acquisition of knowledge, skills, and organizational promotions. In this regard, one of the participants said,

"People view some things as capital such as life and time and they also achieve some things (rewards and benefits). Individuals are like business corporations; they look into how much they spend and what reward they receive. This also holds true for tacit knowledge sharing..., meaning that if we know how much he spends and which things are valuable to him and we give him those things, that person is encouraged to share his knowledge." (p.3, Knowledge management expert)

Most of the participants believed that individuals should be encouraged to share their tacit knowledge through a reward-and-incentive system. The recognition of both material and immaterial reasons of knowledge sharing must be prioritized. To convert knowledge, taking into account the incentives and encouraging the individuals to acquire the knowledge are of great importance. One of the participants stated,

"At the most, 20\% to 30\% of the knowledge created in the organization can be converted into objective knowledge; some of the individuals are not inclined to share their knowledge, which is because of incentives, intellectual property rights or losing power, and some other individuals do not know what knowledge they possess, but still make use of their unknown knowledge. Therefore, in knowledge management, individuals do not share their knowledge to allow the organization to own their knowledge. "(

\section{Managerial support}

Participants stated that organizational conditions, which led to tacit knowledge sharing, require the support of top managers.

\section{Converting tacit to explicit knowledge}

The participants laid emphasis on the collective aspect of tacit knowledge that is transformed into explicit knowledge through externalizations via team conversations, team learning, and social and specialized networking among experts. They believed that the conversion of tacit knowledge to objective knowledge in the organization begins from creating, sharing, and disseminating knowledge. It is here onward that the acquisition and utilization of knowledge follows. Paving the way for the individual to recount the subject on which he was working results in the conversion of tacit knowledge and into the explicit one. With respect to the conversion of tacit knowledge into the explicit, one participant stated,

"The more you minimize the project, the easier you achieve the desired outcome. For example, initially only the tacit knowledge should be converted into the explicit knowledge to allow it to be incorporated into the organizational procedures. It can, then, be converted into different by-laws, regulations, and instructions related to organizational procedures and duties." (p.5, Knowledge management expert)

Participants declared that converting tacit knowledge into explicit knowledge can be done through regulations, rules, flowcharts, instructions, organizational processes, and organizational duties.

Documentation and recording the experiences of experts and pioneers and lessons learned

Identifying key individuals, interviewing experts and recording their experiences, and organizational failures and successes were proposed as solutions for utilizing tacit knowledge. By doing so, valuable knowledge is created, which is not recorded anywhere but everyone is looking for. Preparing a booklet and/or expert guide and recording individuals' knowledge and interests in organizing tacit knowledge, for instance, utilizing yellow pages which record people's expertise and acquired interests outside the organization can be effective. The abilities and skills the in- 
dividuals learn outside the organization, which are not included in their organizational duties can also contribute to utilizing tacit knowledge. One of the participants said,

"We should visit the ministers who led the ministry for at least 4 years and ask them what they really did that brought health to its current position in the country? In a project, we talked to 15 ministers and their key deputies. In these talks, we obtained useful information such as the present health networks and the family physician issue. Each of them prepared a chapter of his/her endeavor; the most important part of it was changed to a book. The book was then changed to a presentable multi-media file. Iran's Primary Health Care Development is one of the best examples in the world." (p.13, Health management and information expert).

\section{Utilizing digital media and virtual electronic space for sharing ideas}

Defining required infrastructures including IT, databases, portals, codifying ideas into thematic categories, and the ability to search with keywords can facilitate tacit knowledge sharing and utilization.

\section{Storytelling}

Storytelling was a proposed mechanism in this respect. Recording stories can contribute to capturing tacit knowledge in the organization. One of the participants said,

"Storytelling is one of the methods of capturing knowledge. Our culture was very rich in this regard, but we are losing this storytelling method. Stories and parables convey one's knowledge to others well; knowledge stays in peoples' minds, but unfortunately, we use this method less in our organizations. There were some attempts before the revolution. One of them was the 'Vital Horoscope'. The inventor, for example, who was a pioneer in this field, narrated the story of the Vital Horoscope; how it was created, where the idea came from, and how it was designed ...." (p.13, Health management and information expert).

\section{Sharing tacit knowledge}

Apprenticeship, mentoring, learning by doing

Utilizing master-apprentice methods and learning by observation lead to gaining experience and extracting knowledge.

\section{Defining the process of tacit knowledge utilization}

The proposed process for utilizing tacit knowledge is a systemic and dynamic form, which includes all dimensions of knowledge sharing, maintenance, extraction, and utilization. In this regard one participant stated,

"We cannot separate knowledge sharing from knowledge maintenance, extraction, and utilization; they should all be considered together. In other words, when we consider sharing, we come to utilization and then to maintenance, etc. ... They are important when considered together and a systemic model should consider all dimensions, just like the hand which is useful as long as it is attached to a total system called the body. Best components don't necessarily make the best system so in designing a system we should pay attention to the suitability of components. It cannot be said that sharing doesn't affect knowledge discovery or creation. They have exchanges like a dynamic system and affect each other." (p.3, Knowledge management expert).

Participants believed that the process of converting tacit to explicit knowledge as a cyclical and repetitive process was possible through the below mentioned themes:

Understanding the existing situation and identifying knowledge points: This, which includes determining organizational processes and points, where tacit knowledge enters into this process.

Identifying knowledge items: It includes lessons learned, meeting agendas, etc. One of the participants referred to these knowledge items and said,

"Knowledge items or lessons learned are those lessons one learns in one's own working field. For instance, the notebook of technical features is a kind of knowledge item. Tender notices, notes, patterns, minutes for agreements, models for selecting a contractor, whether as a checklist or in people's minds, and detailed reports on projects' settlements are all knowledge items." (p.4, Knowledge management expert)

Harvesting knowledge items: This is a continuous process. Among different methods of harvesting, one can refer to writing little essays. One of the participants referred to harvesting knowledge items and said,

"Harvesting knowledge items is like what a farmer does to a plant to make it grow again. It is a continuous process. It can be done through little essays or holding camps, where people's talks are recorded. After recording, some experts classify and categorize these recordings, and its every part is put in the relevant category." (p.4, knowledge management expert).

Assessment, codification, and standardization of harvested knowledge items

Entering standardized knowledge items into organizational processes and knowledge repository and updating: The aforementioned themes are depicted in Diagram 1.

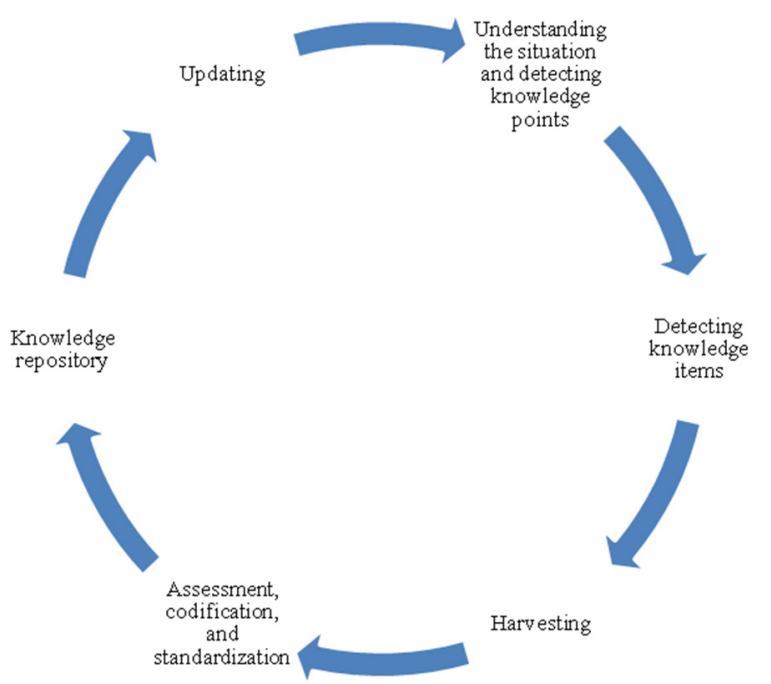

Diagram 1. Process of converting tacit knowledge into explicit knowledge 


\section{Discussion}

In this study, we explored the facilitating factors of knowledge sharing and methods of utilizing tacit knowledge in health organizations from the perspectives of experts in the field. The most prominent facilitating factors, which were mentioned for sharing tacit knowledge, included providing an agile organizational structure, taking it into consideration at the time of workplace evaluation, and creating incentives. In line with our findings, social, organizational, and technological conditions were found to be effective in sharing knowledge in Van Den Brink's study. Depending on the facilitating conditions for sharing knowledge in organizations, they could improve their readiness stage of knowledge dissemination (unawareness, investing and saving knowledge, knowledge route map, taking a common policy, and organizational learning). Organizations that have an open system translate knowledge and are flexible. The organization's environment should provide the context for collaboration, dialogues, and communication among members to create and share knowledge (19).

In our study, more focus is laid on the embedded form of collective tacit knowledge in the organization, when it comes to the dimensions of organizational knowledge. According to the participants, sharing this knowledge requires a dynamic and agile infrastructure based on networks and social capital, and an organizational culture encouraging knowledge, and innovation dissemination. Utilizing tacit knowledge at the organizational level is a different process compared to the group and individual levels, wherein knowledge is captured at the macro level. Malhotra has referred to 4 processes including grafting, vicarious learning, experiential knowledge, and inferential processes of knowledge capture within an organization. The results of this captured knowledge go into the organizational knowledge repository or organizational memory and can be codified (20). Brown and Duguid used the term 'communities of practice for embedded knowledge', which refers to the interactive nature of learning. Societies in which knowledge dissemination occurs create social capital and provide the chance for networking and communication (21, 22).

In a study conducted in China, certain interventions were performed in an organization to enhance the individuals' relations and facilitate translation of tacit knowledge among researchers, which included having intraorganizational and interorganizational workshops and seminars revolving around problem- solving, using supportive tools for a two-sided cooperation, cultural and behavioral changes for encouraging scientific relations between different groups, and using knowledge facilitators. Also, individuals outside the teams were considered important in expressing new ideas, and groups were suggested to hold seminars and invite people from other areas of knowledge. These interventions eventually led to expanding intragroup and intraorganizational relations between individuals. Informal networks play a crucial role in tacit knowledge management and the correct management of these networks leads to effective sharing and translating tacit knowledge (11). These solutions should be applied in health organizations as well. In our study, participants underscored the embodied knowledge of tacit knowledge, which is closer to the empirical knowledge realm of Malhotra's classification and Polanyi's observation and practical experience that does not need to be embodied in the frameworks of conscious decisions (23).

Results revealed that organizational conditions, which lead to tacit knowledge sharing, require the support top managers. In a study conducted among local managers in Queensland, Australia, the effect of leaders' support on tacit knowledge sharing was investigated. The results revealed that top management support had a positive effect on tacit knowledge conversion and translation (9). Managers can play an effective role in this regard by creating justice, trust, and interactive environments such as restaurants, cafes, gyms, and technological infrastructures, and establishing encouraging and motivating processes, and a learning organization (24). This emphasizes the role of managers in health organizations in creating infrastructures and processes that lead to tacit knowledge sharing and utilization.

The process the participants proposed for utilizing tacit knowledge was a systemic and dynamic one. Stewart, Lundvall and Johnson, and Wiig classified tacit knowledge as motion knowledge, knowledge of cosmological rules, understanding systems and schematics, their purpose and cause (22), and thus utilizing this knowledge follows the same rules.

The steps mentioned in the process of converting tacit into explicit knowledge is in line with Dalkir's model (2013), which includes a cycle containing knowledge capture and/or creation, knowledge sharing and dissemination, knowledge acquisition and application, which are assessed between knowledge capture and knowledge dissemination and then is contextualized in a way so that it can be understood and applied. Then, knowledge application provides a feedback for knowledge capture/creation to have the knowledge content updated (22). The process proposed by the participants of this study can be realized when the tacit knowledge capture is possible by making the scopes smaller, determining processes and entry points, and harvesting using several ways such as storytelling, recording experiences, etc.

Participants declared that designing a program to manage and utilize tacit knowledge in an organization demands understanding why this kind of knowledge is not used. Van Den Brink proposes a repeating cycle, which includes the following stages: determining the nature of organizational environment from the perspective of its dynamics; assessing the facilitating conditions of knowledge sharing socially, organizationally, and technologically; the value degree of facilitating conditions and ranking them; and determining the KT stage. Using facilitating conditions suitable for the KT phase of the organization helps the organization to better plan for tacit knowledge sharing (19). This classification can be an effective operational guide in planning knowledge sharing in an organization. Depending on the conditions of health organizations and regardless of their activity and size, the following questions should be clearly 
answered: What is the preparatory phase of the organization for managing this kind of knowledge? What are the enabling conditions which suit these phases? And what potentials are there in the organization in this regard?

Some participants proposed having knowledge workers or an organizational unit to facilitate tacit knowledge exchange in the organization. Some others suggested having a common or intermediary language or translator for extracting knowledge, especially technical and professional knowledge. A study illustrated that choosing top knowledge managers is a way for motivating, guiding, and coordinating a knowledge management plan, which does not probably suffice by itself and is not necessary in all organizations (25).

The results of our study showed that the collective dimension of tacit knowledge converts into explicit knowledge through outsourcing such as team discussions, group learning, community-making and/or social, people, and professional networking between experts and people to find answers to these questions.

Communities of practice are indirectly related to each other. Groups meet each other unofficially and regularly out of the organizational structure. Communal knowledge and its translation lead to innovation. World Bank, for instance, has created $100 \mathrm{~s}$ of such societies to translate knowledge about development (3).

According to the results, when not externally rewarded, members of an organization have no incentive for sharing their hidden knowledge and their experiences. On the contrary, a study by Osterloh and Frey, geology experts, were asked to translate their tacit knowledge and experiences to young engineers. The question was what motivated these experts to translate their knowledge to those young engineers. They believed that external incentives and financial rewards did not affect the extent to which they translated knowledge, nor did it affect the way they did it. However, feeling competent about it satisfied them. In our study, the participants believed that internal incentives had the most impact. Participants were interested in team and group works, which provided the context for translating tacit knowledge. When a beginner faced a problem and asked the expert, it was an opportunity for the expert to think and add to his/her own experience and knowledge. Financial rewards were effective only if they symbolized experts' competencies (26). What is important is that rewards should be fair (3). These contradictory findings may result from cultural differences of different societies.

Participants believed that converting tacit knowledge into explicit knowledge can be done through flowcharts, instructions, organizational processes, and organizational duties. In his study, McKenzie described visual literacy and referred to using flowcharts, diagrams, and graphic symbols in extracting tacit knowledge (27). Participants suggested documentation and recording experiences of experts and pioneers, and lessons learned. Garcia-Perez and Ayres used action research in their study to clarify experts and key figures' experiences and knowledge. The study was conducted in the following phases: the initial phase in which the scope was determined and the team was selected; the preparation phase in which interviews were conducted, concepts were extracted and the first draft was prepared; and the phase in which collaborative modeling sessions were held to introduce and discuss the concepts. Then, modeling was done and goal achievements were evaluated. The method applied in the latter study was assessed as an effective method (28).

One of the models used by NASA (National Aeronautics and Space Administration) for utilizing the lessons learned in the organization set up committees consisting of technical experts, quality assurance organizations, and documentary makers. These committees were asked to investigate the reports of incidents and lessons learned and to rank these lessons and their application in existing and planned projects. Lessons learned were used to improve plans and projects through a process of detection, acquisition, organization, analysis, goal setting, dissemination, utilization, and feedback of experiences $(29,30)$.

Interactive learning environments and environments for storytelling, master-apprentice methods, motivations, and using media for sharing ideas were among other methods the participants suggested for facilitating knowledge sharing and utilization of tacit knowledge. In this regard, Xerox, for instance, has created an environment for sharing knowledge that includes chartrooms, catalogues containing successful experiences, yellow papers, and websites (22). IBM (International Business Machines Corporation) considers storytelling as a powerful tool for discovering knowledge. In this organization, IT has an important role in aggregating and translating tacit knowledge (3). In 2 nonstate-owned companies, which cooperated with the Ministry of Health for treating tuberculosis, tacit knowledge management was done through software and email lists and health internetwork access to research activities in developing countries (31).

Although qualitative and deep understanding of expert opinion on the tacit knowledge issue is the strength of this study, its weakness is the limited generalizability of the results of qualitative research.

\section{Conclusion}

Because tacit knowledge includes $80 \%$ and explicit knowledge includes only $20 \%$ of all knowledge in organizations, methods for tacit knowledge utilization should be appreciated more. Despite the known value of explicit knowledge, the required information cannot be extracted from this knowledge most of the time and tacit knowledge is required. For instance, when we want to know how something is done, we need to look for how it is done and answer with experience. The findings of the current research indicate that health organizations first need to change their structure and performance to obtain the capacity for utilizing this knowledge. Moreover, because the themes extracted in this study are rarely used in Iranian health organizations, the results will be helpful in guiding the development of knowledge utilization strategies and planning in these organizations.

\section{Acknowledgements}

This study was supported by the 'Knowledge Utilization 
Research Center of Tehran University of Medical Sciences (Grant No: 89-04-102-12024). We express our sincere gratitude to Ms Somayeh Faghih Mirzaei from Qom University, Dr. Salarian Zadeh from the Ministry of Health \& Medical Education, Dr. Najarian from the Medical Informatics Department of Deputy of Research and Technology, Mr. Abbas Afrazeh from System Management \& Utilization Department of Amirkabir University, and Ms. Sholeh Arastoopour from Deputy of Research and Technology of the Regional Information Center for Science and Technology for sharing their invaluable ideas.

\section{Conflict of Interests}

The authors declare that they have no competing interests.

\section{References}

1. Kiefer L, Frank J, Di Ruggiero E, Dobbins M, Manuel D, Gully PR, et al. Fostering evidence-based decision-making in Canada. Can J Public Health. 2005;96(3):1-18

2. Lincoln YS, Lynham SA, Guba EG. Paradigmatic controversies, contradictions and emerging confluences. In Handbook of Qualitative Research. 2011

3. Dealtry R, Smith EA. Communities of competence: new resources in the workplace. J Workplace Learn. 2005;17(1/2):7-23.

4. Kothari AR, Bickford JJ, Edwards N, Dobbins MJ, Meyer M. Uncovering tacit knowledge: a pilot study to broaden the concept of knowledge in knowledge translation. BMC Health Serv Res. 2011;11(1):198.

5. Braude HD. Clinical intuition versus statistics: different modes of tacit knowledge in clinical epidemiology and evidence-based medicine. Theor Med Bioeth. 2009;30(3):181-98.

6. Mahmood A, Qureshi M, Shahbaz Q. An examination of the quality of tacit knowledge sharing through the theory of reasoned action. J Qual Tech Manag. 2011;7(1):39-55.

7. Yakhlef A, Sié L, Julen P. The effects of rewards on the motivation of experts to transfer their knowledge. In: Proceedings of the 8th EURAM (European Academy of Management) Conference, Ljubljana and Bled, Slovenia. Citeseer; 2009.

8. Nonaka I, Von Krogh G. Perspective-Tacit knowledge and knowledge conversion: Controversy and advancement in organizational knowledge creation theory. Organ Sci. 2009;20(3):635-52.

9. Rowe PA, Christie MJ. Civic entrepreneurship in Australia: Opening the "black box" of tacit knowledge in local government top management teams. IJPSM. 2008;21(5):509-24.

10. Janson A, McQueen RJ. Capturing leadership tacit knowledge in conversations with leaders. Leader Organ Dev J. 2007;28(7):646-63.

11. Nie K, Lin S, Ma T, Nakamori Y. Connecting informal networks to management of tacit knowledge. J Syst Sci Syst Eng. 2010;19(2):23753.

12. Greenhalgh J, Flynn R, Long AF, Tyson S. Tacit and encoded knowledge in the use of standardised outcome measures in multidisciplinary team decision making: A case study of in-patient neurorehabilitation. Soc Sci Med. 2008;67(1):183-94.

13. Smith A, Goodwin D, Mort M, Pope C. Expertise in practice: an ethnographic study exploring acquisition and use of knowledge in anaesthesia. Br J Anaesth. 2003;91(3):319-28.

14. Büssing A, Herbig B, Latzel A. Knowledge-Experience-Action. In D. Frey, H. Mandl \& L. von Rosenstiel (Eds.), Knowledge and action, Cambridge, MA: Hogrefe \& Huber Publishers; 2006:175.

15. Yoshioka-Maeda K, Murashima S, Asahara K. Tacit knowledge of public health nurses in identifying community health problems and need for new services: a case study. Int J Nurs Stud. 2006;43(7):819-26.

16. Gabbay J, le May A. Evidence based guidelines or collectively constructed 'mindlines?' ethnographic study of knowledge management in primary care. BMJ. 2004;329(7473):1013-8.

17. Friedman LH, Bernell SL. The importance of team level tacit knowledge and related characteristics of high-performing health care teams. Health Care Manage Rev. 2006;31(3):223-30.

18. Gelfand MJ, Erez M, Aycan Z. Cross-cultural organizational behavior. Annu Rev Psychol, 2007;58:479-514.

19. Van den Brink D, Odekerken-Schröder G, Pauwels P. The effect of strategic and tactical cause-related marketing on consumers' brand loyalty. J Consum Mark. 2006;23(1):15-25.

20. Malhotra Y. Knowledge management and virtual organizations: USA, IGI Publishing Hershey; 2000.

21. Brown JS, Duguid P. Balancing act: how to capture knowledge without killing it. Harv Bus Rev. 2000;78(3):73-80.

22. Dalkir K. Knowledge management in theory and practice: Routledge; 2013.

23. Lam A. Lambermont-Ford JP. Knowledge sharing in organisational contexts: a motivation-based perspective. J Knowl Manag. 2010;14(1):51-66.

24. Song D. The tacit knowledge-sharing strategy analysis in the project work. Intl Bus Res. 2009;2(1):83.

25. Berkes F. Evolution of co-management: role of knowledge generation, bridging organizations and social learning. J. Environ. Manage. 2009; 90(5):1692-1702

26. Osterloh M, Frey BS. Shareholders should welcome knowledge workers as directors. J Manag Gov. 2006;10(3):325-345.

27. McKenzie B. Flowcharting and Visual Literacy: Exploring Tacit Knowledge through the Visual; 2008.

28. Garcia-Perez A, Ayres R. (eds). Collaborative Development of Knowledge Representations-A Novel Approach to Knowledge Elicitation and Transfer. In: The 9th European Conference on Knowledge Management: Eckm, Academic Conferences Limited; 2008.

29. Olla P, Holm J. The role of knowledge management in the space industry: important or superfluous? J Knowl Manag. 2006;10(2):3-7.

30. Hooey BL, Foyle DC. (eds). Requirements for a design rationale capture tool to support NASA's complex systems. International Workshop on Managing Knowledge for Space Missions Pasadena; 2007.

31. Kebede G. Knowledge management: An information science perspective. Int J Inf Manage. 2010;30(5):416-424. 